\title{
Kinetic energy efficiency of single ventricle and TCPC using 4D flow MRI
}

\author{
Alejandro Roldán-Alzate ${ }^{*}$, Sylvana García-Rodríguez ${ }^{1}$, Petros V Anagnostopoulos², Shardha Srinivasan², \\ Christopher J Francois ${ }^{1}$ \\ From 18th Annual SCMR Scientific Sessions \\ Nice, France. 4-7 February 2015
}

\section{Background}

Altered hemodynamics in total cavopulmonary connection (TCPC), a palliation of single ventricle defects, results in long-term complications, such as decreased exercise capacity, arrhythmia, and ventricular failure [1]. Non-invasive hemodynamic evaluation of TCPC has been an important clinical challenge. Several studies have tried to understand and predict specific flow features using a combination of patient-specific MRI data and computational tools to develop more realistic numerical and physical models. Most numerical studies have based their analyses of TCPC efficiency on energy loss calculations, but assumptions such as rigid walls and idealized flow conditions might affect accuracy hindering clinical applicability [2]. The purpose of this study was to calculate kinetic energy (KE) from in vivo 4D Flow MRI velocity measurements, in the TCPC and single ventricle for assessing efficiency of the system.

\section{Methods}

4D Flow MRI was performed in 6 TCPC patients and 6 healthy subjects following an IRB-approved protocol. 4D flow MRI (PC VIPR) imaging parameters were: imaging volume: $32 \times 32 \times 24 \mathrm{~cm}, 1.25 \mathrm{~mm}$ acquired isotropic spatial resolution, TR $/ \mathrm{TE}=6.4 / 2.2 \mathrm{~ms}$, VENC $=200 \mathrm{~cm} / \mathrm{s}$ (extracardiac) and $100 \mathrm{~cm} / \mathrm{s}$ (atrio-pulmonary) [3]. Vessel segmentation was performed (MIMICs, Materialise, Leuven, Belgium) from PC angiograms; visualization and quantification were performed in EnSight (CEI, Apex, NC). Two calculations were considered: 1 ) the energy efficiency of the TCPC, and thus in the pulmonary circulation, and 2) the energy efficiency of the systemic ventricle, thus evaluating the systemic circulation. For

${ }^{1}$ Radiology, University of Wisconsin, Madison, WI, USA

Full list of author information is available at the end of the article the first, $\mathrm{KE}_{\text {in }}\left(\mathrm{KE}_{\mathrm{IVC}}+\mathrm{KE}_{\mathrm{SVC}}\right)$ was compared to $\mathrm{KE}_{\text {out }}$ $\left(\mathrm{KE}_{\mathrm{RPA}}+\mathrm{KE}_{\mathrm{LPA}}\right)$ as \%KE loss $=\left(\mathrm{KE}_{\text {in }}-\mathrm{KE}_{\text {out }}\right) / \mathrm{KE}_{\text {in }}$. For the second calculation, ventricular energy efficiency was defined as the ratio of aortic flow and systemic ventricular $\mathrm{KE}\left(\mathrm{Q}_{\mathrm{Ao}} / \mathrm{KE}_{\mathrm{SV}}\right)$ (Figure 1$)$.

\section{Results}

Kinetic energy loss in the TCPC was $31 \pm 20 \%$. Figure 2 compares systemic efficiency in TCPC and healthy subjects. Systemic efficiency was lower in TCPC patients (7.6 $\pm 6.1(\mathrm{ml} / \mathrm{s}) / \mathrm{mJ})$ than that in healthy volunteers $(63 \pm 17$ $(\mathrm{ml} / \mathrm{s}) / \mathrm{mJ}))$. Within the TCPC group, patients with single right ventricle had higher $(9.5 \pm 8.5(\mathrm{ml} / \mathrm{s}) / \mathrm{mJ})$ that those with left single ventricle $(5.2 \pm 2.2(\mathrm{ml} / \mathrm{s}) / \mathrm{mJ})$.

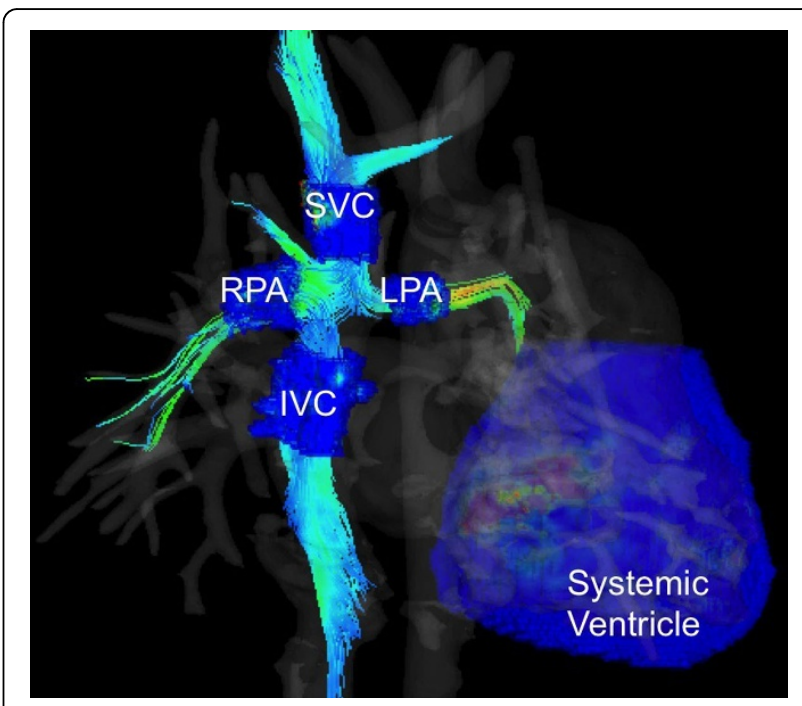

Figure 1 Visualization of flow and kinetic energy in an extracardiac TCPC and its systemic ventricle obtained from 4D flow MRI. 


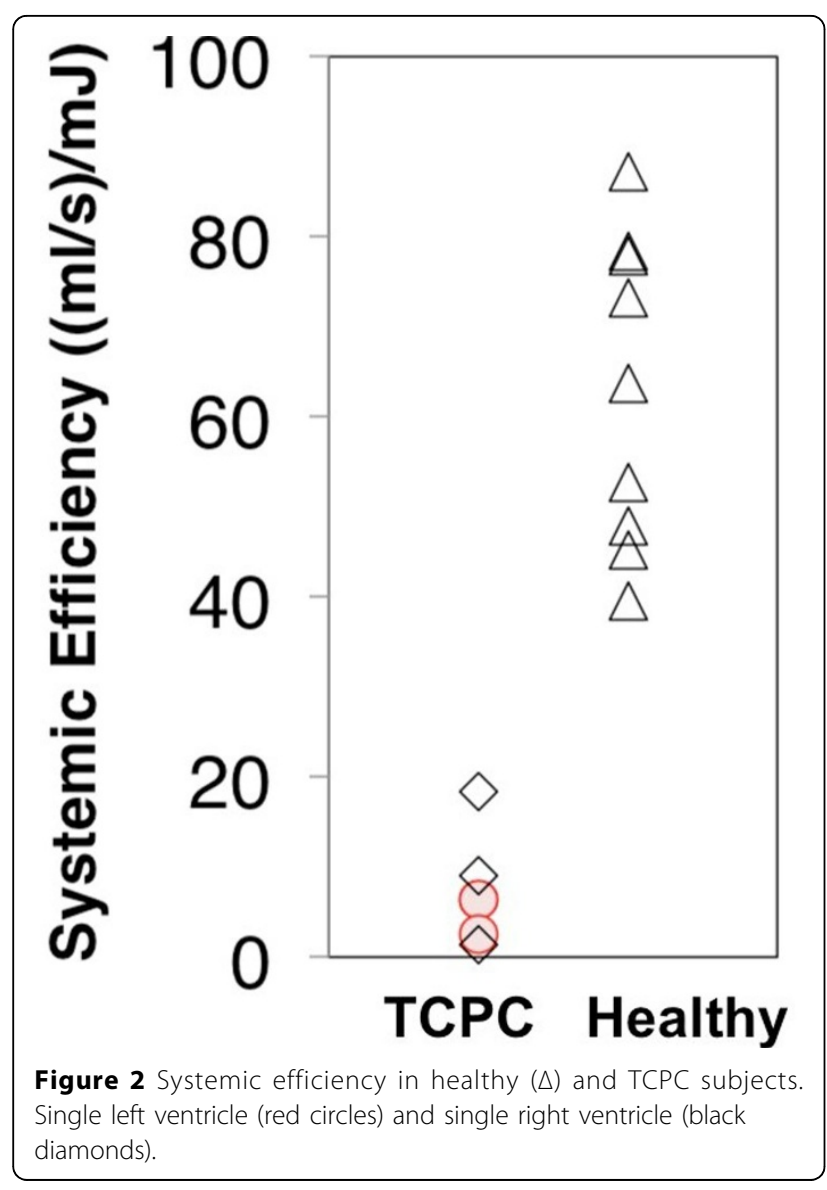

\section{Conclusions}

As expected, TCPC patients had less systemic efficiency; however, high variability was observed in both groups suggesting the need for larger sample studies. Successful implementation of in vivo 4D Flow MRI provides a powerful non-invasive surveillance tool that has the potential to allow the clinician to follow the performance of TCPC over time, identify potential hemodynamic deterioration and intervene in an asymptomatic phase to increase the life span of the operation.

\section{Funding}

American Heart Association - Scientist Development Grant (Alejandro Roldán-Alzate).

\section{Authors' details}

${ }^{1}$ Radiology, University of Wisconsin, Madison, WI, USA. ${ }^{2}$ Cardio-Thoracic

Surgery, University of Wisconsin, Madison, WI, USA. ${ }^{3}$ Pediatric Cardiology,

University of Wisconsin, Madison, WI, USA.

Published: 3 February 2015

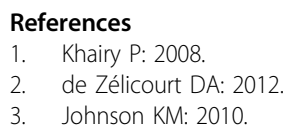

3. Johnson KM: 2010.

doi:10.1186/1532-429X-17-S1-097

Cite this article as: Roldán-Alzate et al:: Kinetic energy efficiency of single ventricle and TCPC using 4D flow MRI. Journal of Cardiovascular Magnetic Resonance 2015 17(Suppl 1):Q97.
Submit your next manuscript to BioMed Central and take full advantage of:

- Convenient online submission

- Thorough peer review

- No space constraints or color figure charges

- Immediate publication on acceptance

- Inclusion in PubMed, CAS, Scopus and Google Scholar

- Research which is freely available for redistribution 